\title{
Two level undercut-profile substrate-based filamentary coated conductors produced using metal organic chemical vapor deposition
}

\section{Insinga, Andrea R.; Sundaram, Aarthi; Hazelton, Drew W.; Rodriguez Zermeno, Victor Manuel ; Abrahamsen, Asger Bech Bech; Opata, Yuri Aparecido; Grivel, Jean-Claude; Lundeman, Jesper Holm; Ryming, Alexander Engels; Schweer-Gori, Frederik}

Total number of authors:

11

\section{Published in:}

I E E E Transactions on Applied Superconductivity

Link to article, DOI:

10.1109/TASC.2018.2808365

Publication date:

2018

Document Version

Peer reviewed version

Link back to DTU Orbit

Citation (APA):

Insinga, A. R., Sundaram, A., Hazelton, D. W., Rodriguez Zermeno, V. M., Abrahamsen, A. B. B., Opata, Y. A. Grivel, J-C., Lundeman, J. H., Ryming, A. E., Schweer-Gori, F., \& Wulff, A. C. (2018). Two level undercut-profile substrate-based filamentary coated conductors produced using metal organic chemical vapor deposition. I $E E$ Transactions on Applied Superconductivity, 28(4), [6601705]. https://doi.org/10.1109/TASC.2018.2808365

\section{General rights}

Copyright and moral rights for the publications made accessible in the public portal are retained by the authors and/or other copyright owners and it is a condition of accessing publications that users recognise and abide by the legal requirements associated with these rights.

- Users may download and print one copy of any publication from the public portal for the purpose of private study or research.

- You may not further distribute the material or use it for any profit-making activity or commercial gain

- You may freely distribute the URL identifying the publication in the public portal 


\title{
Two level undercut-profile substrate-based filamentary coated conductors produced using metal organic chemical vapor deposition
}

\author{
A. R. Insinga, A. Sundaram, D. W. Hazelton, V. M. R. Zermeno, A. B. Abrahamsen, Y. A. Opata, J.-C. Grivel, \\ J. H. Lundeman, A. E. Ryming, F. Schweer-Gori, and A. C. Wulff
}

\begin{abstract}
The two level undercut-profile substrate (2LUPS) has been introduced as a concept for subdividing rare-earth- $\mathrm{Ba}_{2} \mathrm{Cu}_{3} \mathrm{O}_{7}$ coated conductors $(\mathrm{CC})$ into narrow filaments that effectively reduces the AC losses and improves field stability for DC magnets. The 2LUPS consists of two levels of plateaus connected by a wall with an undercut-profile, which enables a physical separation of the superconducting layer between the plateaus without reducing the effective width of the superconducting layer.

In this study we report for the first time the results of fabrication and characterization of a filamentary $\mathrm{CC}$ produced in an industrial setup by SuperPower Inc. using ion beam assisted deposition and metal organic chemical vapor deposition (IBAD-MOCVD) on a 2LUPS realized at the Technical University of Denmark (DTU), whereas previous studies discussed the fabrication using alternating beam assisted deposition and pulsed laser deposition (ABADPLD).

We also present Hall probe scanning measurements performed using a standard THEVA TAPESTAR ${ }^{\text {TM }}$ XL machine that is routinely employed for industrial critical current characterization of long length CCs. From these results is it clear that additional analysis of the measured field profiles are required when characterizing filamentary 2LUPS CC using a standard TAPESTAR ${ }^{\text {TM }}$ setting. Using a model representation of the 2LUPS we calculated the expected magnetization response by means of finite element methods simulations and we find a good agreement with the experimentally observed magnetic profiles.
\end{abstract}

Index Terms-Multifilamentary superconductors, Hightemperature superconductors, Magnetic variables measurement, Finite element analysis.

\section{INTRODUCTION}

$\mathbf{H}$ IGH-TEMPERATURE SUPERCONDUCTORS (HTS) are relevant to many scientific and technological applications [1]-[3] such as electric motors and generators, electric power distribution systems, and high-field electromagnets.

Manuscript received October 9, 2012. This work was supported in part by the Danish Council for Independent Research (ATOMIS project ID DFF - 611100252).

A. R. Insinga, A. C. Wulff, Y. A. Opata, J.-C. Grivel, Alexander E. Ryming, and F. Schweer-Gori are with the Department of Energy Conversion and Storage, Technical University of Denmark, 4000 Roskilde, Denmark.

A. Sundaram and D. W. Hazelton are with SuperPower, Inc., Schenectady, NY 12304, USA.

V. M. R. Zermeno is with Institute for Technical Physics, Karlsruhe Institute of Technology, Karlsruhe, Germany.

A. B. Abrahamsen is with the Department of Wind Energy, Technical University of Denmark, 4000 Roskilde, Denmark.

J. H. Lundeman is with SUBRA IVS, 2765 Smørum, Denmark.

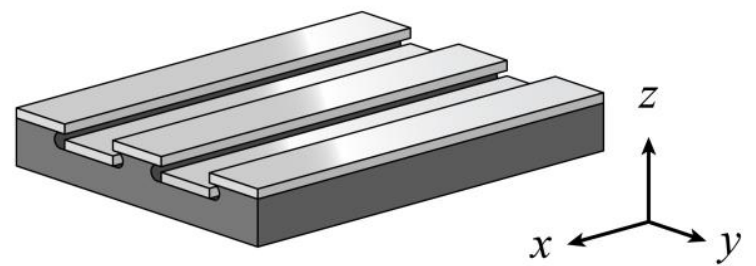

Fig. 1. Illustration of the geometry of 2LUPS-based CC. The dark gray and light gray regions indicate the substrate and the SC layer, respectively.

Superconducting electromagnets are used in magnetic resonance imaging (MRI) and nuclear magnetic resonance (NMR) devices, particle accelerators, and many other scientific instruments and in general made using low temperature superconductors (LTS). HTS present several advantages over LTS which include higher irreversibility fields [4] and higher operation temperatures. Rare-Earth Barium-Copper-Oxides, such as $\mathrm{YBa}_{2} \mathrm{Cu}_{3} \mathrm{O}_{7}$ and $\mathrm{Gd}_{1-\mathrm{x}} \mathrm{Y}_{\mathrm{x}} \mathrm{Ba}_{2} \mathrm{Cu}_{3} \mathrm{O}_{7}$ (GYBCO), are a welldocumented [1]-[4] class of HTS materials in the $2^{\text {nd }}$ generation HTS wires, i.e. called coated conductors (CC). The width of these tapes is an important design parameter, which affects hysteretic losses in AC applications as well as the magnetic field drift in strong DC magnets [5]-[7]. These effects are caused by the screening current, which arises inside the superconductor tape to prevent the magnetic field from penetrating the material. This current and its variation over time are responsible for AC-losses and may also reduce the precision of the required magnetic field distribution, which is critical for many superconducting magnet applications. It has previously been shown that these detrimental effects can be mitigated by transposing the tape and subdividing the tape along its width into many decoupled filaments [7], [8]. Filamentation methods employed to achieve this subdivision include mechanical striation, laser striation and ink jet printing of filaments [9]-[12]. The challenge with these approaches comes from the reduction of the effective width of the superconducting (SC) layer that decreases the engineering critical current density, in addition to potential critical damage over the long length. Filamentization can potentially be achieved without reduction of the effective width of the tape using the two level undercut profile substrate (2LUPS) concept [13], [14] (see Fig. 1), consisting of two levels of plateaus in the metal substrate surface separated by a vertical displacement with an undercut profile. 
The undercut profile can be realized by surface modification of the areas of the substrate corresponding to the lower plateaus by means of electrochemical etching. In this study we present, for the first time, experimental characterization of a 2LUPS CC produced by SuperPower Inc. using ion beam assisted deposition and metal organic chemical vapor deposition (IBAD-MOCVD) in a commercial system on a 2LUPS prepared at the Technical University of Denmark. No posttreatment was conducted after layer depositions. The performance of the 2LUPS CC was evaluated by means of Hall probe scanning using a Theva TAPESTAR ${ }^{\mathrm{TM}} \mathrm{XL}$ machine that is routinely employed for analysis of long length samples at SuperPower Inc. and compared to a CC produced without surface profiles. The 2LUPS CC is evaluated in this system since filamentary long length samples would eventually also require such industrial characterization. However, data obtained using a TAPESTAR ${ }^{\mathrm{TM}}$ machine does not take into account geometrical effects and further interpretation is therefore required to evaluate the critical current. In order to correctly calculate the critical current of the filamentary 2LUPS CC, we compare the experimental data with computations performed using FEM analysis.

\section{METHODS}

\section{A. Samples fabrication}

Starting from a commercial Hastelloy C276 tape $(L \times W \times T=300 \mathrm{~mm} \times 10 \mathrm{~mm} \times 0.89 \mathrm{~mm})$, wet electrochemical etching is employed in order to create the desired 2LUPS geometrical profile and to reduce the surface roughness [14].

Protective masking tape has been applied and cut in a reelto-reel system after which 3 strips were peeled off. Lower plateaus were then formed during subsequent etching. The widths of each of the two lateral filaments are $2.8 \pm 0.1 \mathrm{~mm}$, while the five central plateaus has a width of $0.9 \pm 0.1 \mathrm{~mm}$. The reference sample was produced using the standard etching procedure [14]. Following the 2LUPS fabrication, the buffer and superconducting GYBCO layers are deposited on the substrate in a commercial production system at SuperPower Inc. by means of IBAD-MOCVD [15]. The total thickness of the buffer layers is around $0.2 \mu \mathrm{m}$ and the superconducting $\mathrm{Gd}_{1 \text { - }}$ ${ }_{x} \mathrm{Y}_{\mathrm{x}} \mathrm{Ba}_{2} \mathrm{Cu}_{3} \mathrm{O}_{7}$ layer has a thickness of $1.1 \mu \mathrm{m}$. Finally, a $0.7 \mu \mathrm{m}$ thick protective silver layer is deposited on the GYB$\mathrm{CO}$ layer by means of sputtering.

\section{B. Scanning electron microscopy}

The cross-section of the 2LUPS sample has been analyzed using focused ion beam scanning electron microscopy (FIBSEM) in a Carl Zeiss $1540 \mathrm{XB}$ electron microscope. Fig. 2 shows the cross-section of the 2LUPS and the different layers of the CC. A gap is present in the GYBCO layer just below

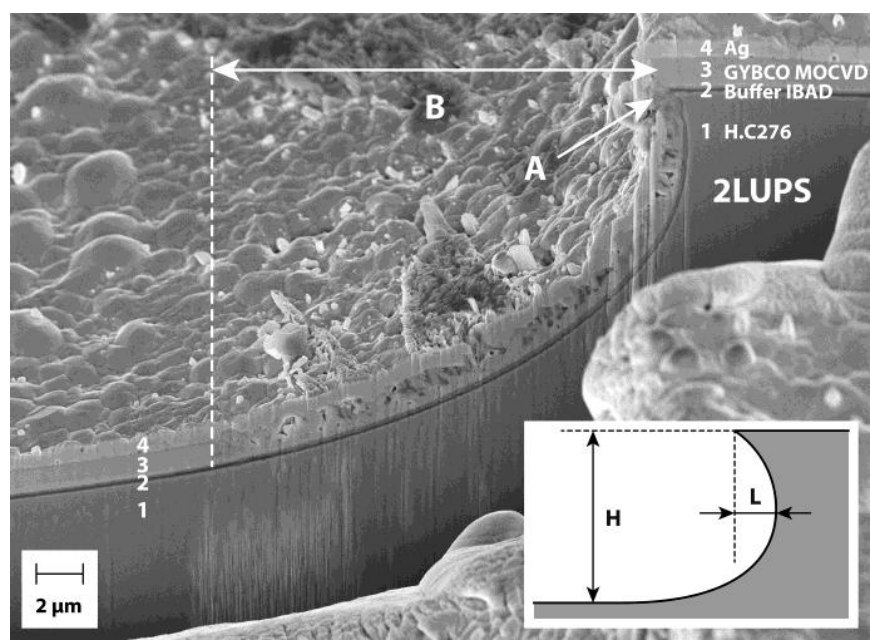

Fig. 2 SEM picture of the cross section of the 2LUPS CC. The SC layer presents a gap, marked by the letter A, and the upper plateau is physically decoupled from the lower plateau. The extent of the region where the $\mathrm{SC}$ is potentially damaged, $\approx 18 \mu \mathrm{m}$, is indicated by the letter B. Numbers $1-4$ mark the different layers: 2LUPS (1), buffer stack (2), Gd-Y-B-CO (3) and $\mathrm{Ag}$ (4). The inset defines the undercut length $L$, and the depth $H$ of the lower plateau.

the edge of the upper plateau (marked by "A") that verifies the desired physical decoupling between the superconducting filaments. It was observed that the undercut length $L=1.2 \pm 0.5 \mu \mathrm{m}$, which is significantly greater, compared to a previously reported Hastelloy-based 2LUPS sample $(L=0.3 \mu \mathrm{m})$ [14]. In the curved region of the lower plateau (see Fig.2) the GYBCO layer is observed to be both porous and irregular, and as such considered non-functional. In contrast, a very dense and homogeneous microstructure is observed on the regions of the plateaus not located in the immediate vicinity of the edge of the filaments, which is typical for $\mathrm{CC}$ production at SuperPower Inc. Measuring several crosssections, it was observed that the extension of the damaged region, marked by " $\mathrm{B}$ ", is equal to $18 \pm 2 \mu \mathrm{m}$, which is significantly smaller than that obtained using other filamentization techniques.

\section{Hall probe scanning}

The remanent magnetic field in different positions along the $x$ direction of the 2LUPS and the reference samples were measured at $77 \mathrm{~K}$ using a TAPESTAR ${ }^{\mathrm{TM}} \mathrm{XL}$ machine at $\mathrm{Su}-$ perPower Inc. The apparatus employs an array of 21 Hall sensors spanning a width of $12 \mathrm{~mm}$ [16]. Fig.3(a) displays the field profile plotted as function of $y$. The data corresponding to the 2LUPS and the reference sample are plotted as red and blue dots, respectively. The error bars shown in the figure correspond to the variation of the field profile along the length $x$ of the tape. The solid lines of matching colors correspond to FEM calculations discussed in the next section. The vertical gray lines indicate the filaments edges of the 2LUPS tape. 
The TAPESTAR ${ }^{\mathrm{TM}}$ software utilizes conventional inversion techniques to compute $I_{C}$ from the magnetic field it generates in the immediate vicinity of the surface of the tape [18]. The resulting plots of the critical current $I_{C}$ as function of the position along the length of the tape, i.e. $x$ direction, are shown in Fig. 4 as dashed lines. It is observed that the critical current of the 2LUPS is substantially lower than that of the reference sample. However, if the spatial resolution is too coarse or the distance of the Hall sensor from the tape is too large to resolve the small signal generated by the narrow filaments, one would expect a similar reduction in the calculated $I_{C}$, which does not correspond to the true value. It is clear that the conventional inversion requires special attention due to the filament geometry. In addition, the setup is not ideal for a complete characterization of filamentized samples and potentially in future studies one would modify the TAPESTAR ${ }^{\text {TM }}$ machine. Taking into consideration the great effect of the distance on the obtained field profiles [19]-[23], we calculated a set of typical field profiles from analytical formulas [17], which can be seen in Fig. 5. The formula, reported in the reference, is derived from the two-dimensional Biot-Savart law applied to a mono-

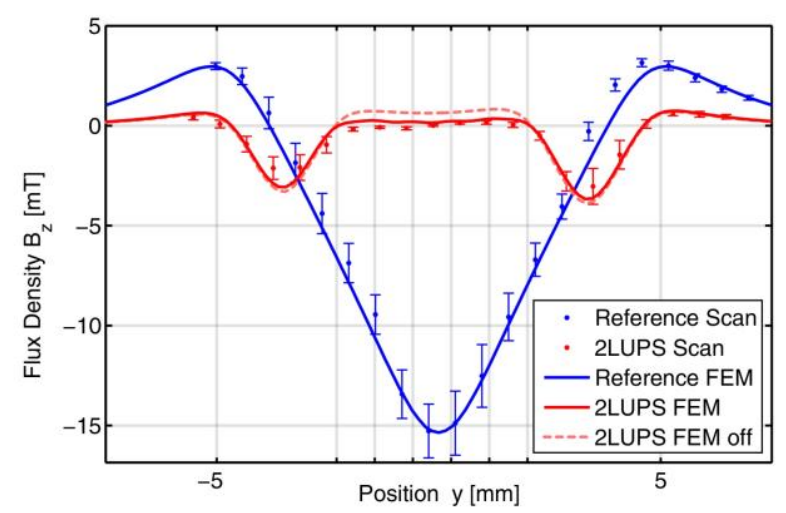

Fig. 3 Hall-probe scanning data obtained at $77 \mathrm{~K}$. The figure shows the distribution as function of $y$ of the magnetic flux density $z$ component. The reference and 2LUPS data are plotted using blue and red colors, respectively. The experimental data is shown as points with error bars, the results of the FEM calculation are shown as solid lines, or as a dashed line for the case of a filamentary tape where only the lateral wider filaments are active. The vertical lines indicate the filaments edges.

dimensional current distribution. The different lines shown in the figure correspond to different values of the distance $\Delta z$ between the Hall sensors and the SC layer of the tapes. The distance $\Delta z$ increases from $0.5 \mathrm{~mm}$ to $1.5 \mathrm{~mm}$ with steps of $0.5 \mathrm{~mm}$, as shown in the figure. As can be noticed, at a distance of $1 \mathrm{~mm}$ from the SC layer the field oscillations generated by the narrow filaments of the central region of the tape are completely smoothed out and would not be resolved by the measurement. The black dashed line corresponds to the case where the narrow filaments are not working. As can be noticed, a significantly higher level in the center would have been expected if the filaments were not working. We performed additional investigations employing finite element method analysis. As discussed in the next section, the field profiles calculated by means of FEM simulation are more realistic than the ones provided by the analytical formula since the

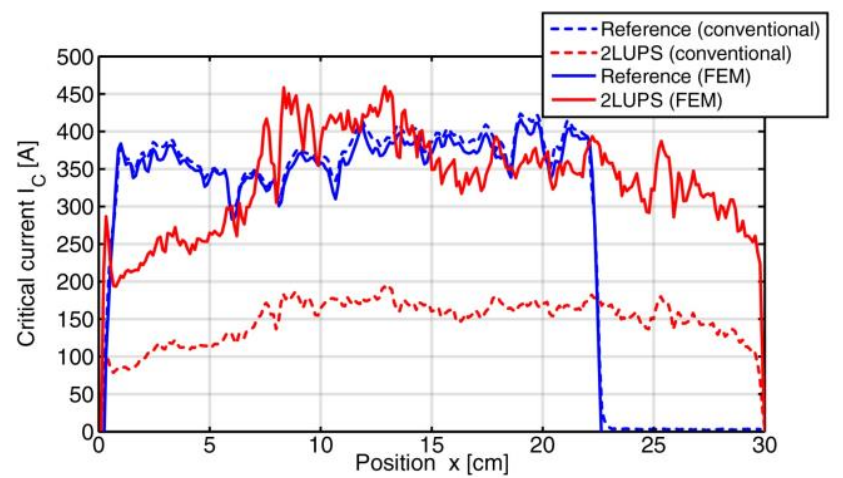

Fig. 4 Calculation of the critical current $I_{C}$ for different positions along the length of the tape, i.e. $x$ direction. The calculations performed using the conventional approach are plotted as dashed lines. The result for the reference sample is consistent with the expected average value of $I_{C}=387 \mathrm{~A}$, whereas for the 2LUPS $I_{C}$ is unrealistically low, indicating that the conventional inversion technique cannot be accurately applied to tapes presenting narrow filaments if the distance between the Hall sensors and the tape is too large. The values of $I_{C}$ computed from the FEM simulations are plotted as solid lines, and for the 2LUPS sample show a better agreement with the expected

FEM simulation solves the partial differential equation governing the magnetic field distribution, while imposing the constraint on the time-dependence of the applied field and the relevant constitutive relations.

\section{Finite Element Method analysis}

The experimental data set has been compared to corresponding results computed by FEM analysis. The computations have been performed using the magnetic field formulation interface of the commercial software COMSOL Multiphysics. The model solves Maxwell's equations and the relevant constitutive relation between the magnetic field $\boldsymbol{H}$ and magnetic flux density $\boldsymbol{B}$. For this study we assumed the following constitutive relation: $\boldsymbol{B}=\mu_{0} \boldsymbol{H}$. A power law $\boldsymbol{J}-\boldsymbol{E}$ constitutive relation is assumed between the electrical field $\boldsymbol{E}$ and the electrical current density $\boldsymbol{J}$ [25], [28]. The relation is parametrized by the critical current density $J_{C}$ :

$$
\boldsymbol{E}=E_{C}\left\|\boldsymbol{J} / J_{C}\right\|^{\mathrm{n}} \boldsymbol{J} /\|\boldsymbol{J}\|
$$

For large values of the exponent $n$, the highly non-linear $\boldsymbol{J}$ $\boldsymbol{E}$ relation practically constrains the current density in each point of space to be either zero or equal to $J_{C}$, thus reproduc-

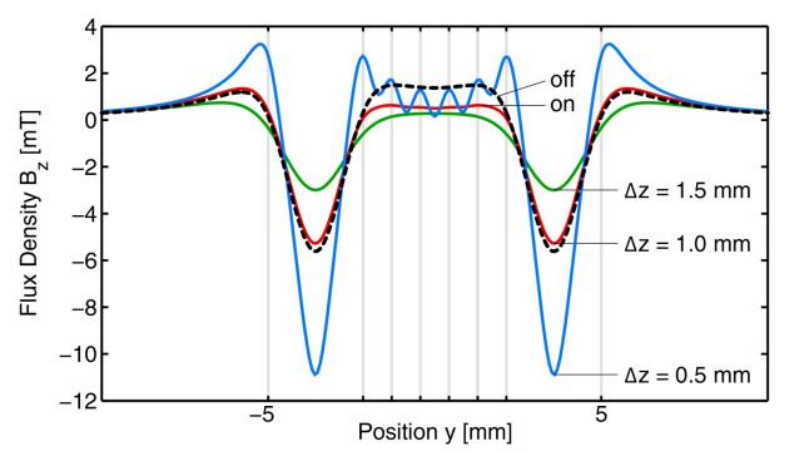

Fig. 5 Field profiles calculated with analytical techniques for different distances $\Delta z$ between the Hall sensors and the 2LUPS tape. The values of $\Delta z$ are indicated in the figure. At a distance of $1 \mathrm{~mm}$ the field oscillations produced by the narrow filaments are not distinguishable. However, the field profile is different when the narrow filaments are not active (black dashed line). 
ing the behavior described by Bean's critical state model [26], [27]. The critical current density $J_{C}$ depends on the norm $B$ of the magnetic flux density. We assume the $J_{C}(B)$ dependence reported in Ref. [24], which has been measured from CCs produced at SuperPower Inc. using the same procedure as for the samples studied in this work and the $J_{C}(B)$ relation can be described by:

$$
J_{C}(B)=J_{C 0} /\left(1+\left(B / B_{0}\right)\right)^{\alpha}
$$

We used the method of least squares to fit this model function to the data reported in Ref. [24], and we obtained the following parameters: $J_{C O}=3.52 \mathrm{MA} / \mathrm{cm}^{2}, B_{0}=3.54 \mathrm{~T}, \alpha=3.62$, assuming that the critical current density $J_{C}$ depends only on the magnitude of $\boldsymbol{B}$. The simulations have been performed using a time-dependent two-dimensional model, which does not resolve the $x$ direction. This simplification is justified since both the samples (2LUPS and flat reference) have length greater than $20 \mathrm{~cm}$, and width of $1 \mathrm{~cm}$. The intensity of the magnetic field applied in the $z$ direction is ramped from $0 \mathrm{mT}$ to $0.037 \mathrm{mT}$, simulating the TAPESTAR ${ }^{\mathrm{TM}}$ measurement. The geometrical characteristics of the reference and 2LUPS samples have been used for the simulation, where the latter also includes the exact width of each of the filaments. Since the actual distance between the SC layer and the Hall sensor is not known with high precision, we calculated the distance by fitting the FEM results to the experimental data. Employing this procedure we obtained the value of $\Delta z=1.19 \mathrm{~mm}$ for the reference sample and $\Delta z=0.85 \mathrm{~mm}$ for the 2LUPS sample, which are values of distance within the expected range.

The results of the FEM computations are shown in Fig. 3 as red and blue solid lines for the 2LUPS and reference sample, respectively. The dashed red line corresponds to the FEM simulation for a tape where the narrow filaments are not working (labelled as "off"). As can be seen, the match between the computed field profiles and the experimental data sets are very good. The relative error $\delta$ between the measured field profile, $B_{E x p}$, and the one calculated from the simulation, $B_{S i m}$, has been quantified using the normalized sum of the squared residuals, expressed by the following formula:

$$
\delta=\left(\sum_{k}\left(B_{E x p}-B_{S i m}\right)^{2}\right) /\left(\sum_{k}\left(B_{E x p}\right)^{2}\right)
$$

Here the index $k$ runs over the 21 experimental data-points. Once expressed in percentage, this formula gives the following result for the reference and 2LUPS samples:

$$
\begin{aligned}
& \delta=0.8 \%, \quad \text { (Reference) } \\
& \delta=6.8 \%, \quad \text { (2LUPS) } \\
& \delta=18.8 \%, \quad \text { (2LUPS - central filaments "Off”) }
\end{aligned}
$$

This agreement indicates that the critical current at zero field $J_{C O}=3.52 \mathrm{MA} / \mathrm{cm}^{2}$, obtained from the data reported in Ref. [24], is consistent with the experimental observation for both the samples. Moreover, the experimental data show a significantly better agreement $(\sim 2.5 \times$ lower relative error $\delta)$ with the simulation which includes the narrow filaments of the central region. For the geometry of the samples under consideration the current density of $3.52 \mathrm{MA} / \mathrm{cm}^{2}$ corresponds to the critical current $I_{C}=387 \mathrm{~A}$, which matches well with the calculation performed with the conventional approach when applied to the reference tape $(375 \pm 27 \mathrm{~A})$. Measurement of $I_{C}$ for the 2LUPS sample using the standard Hall scan setup and employing the conventional conversion, i.e. using the same parameters as for the reference sample, results in much lower $I_{C}$ values (dashed red line in Fig. 4). It is already known that a more careful Hall scan analysis is necessary for CCs presenting narrow filaments as shown in [29]. For this reason we recalculated the value of $I_{C}$ for each position $x$ along the length of the tapes, by considering the scale-factor which would give the best match with the field profile computed by FEM simulation. The results of this calculation are shown in Fig. 4 as solid lines. It can be noticed that our method leads to approximately the same result when applied to the reference tape. For the 2LUPS tape our approach is in much better agreement with the expected value of critical current than the conventional calculation. This indicates that the TAPESTAR ${ }^{\mathrm{TM}}$ setup can be used to interpret functionality of the 2LUPS filaments though a lower working distance between sample and sensor should, and if possible more sensors, should be applied for future characterization to enable a more precise $I_{C}$ evaluation.

\section{CONCLUSION}

A new type of 2LUPS-based filamentary CCs was produced using Ion Beam Assisted Deposition and Metal Organic Vapor Deposition in commercial systems at SuperPower Inc. Microstructural characterization of the GYBCO superconducting layer cross-sections revealed that narrow non-functional regions are present between the upper and lower plateaus. In addition, individual filaments were also found to be physically decoupled when producing the superconducting layer using the large-scale MOCVD technique.

Hall-probe scanning using a commercial Theva TAPES$\mathrm{TAR}^{\mathrm{TM}}$ system, suitable for long length characterization of standard CCs, was employed for critical current measurements of samples. The standard parameters and conventional (magnetic field/current) conversion used in this commercial characterization system are not ideal for evaluation of filamentary CCs and significantly lower $I_{C}$ values were observed for the 2LUPS CC compared to a standard flat sample. We performed FEM computations taking into consideration the 2LUPS geometry and the expected $J_{C}(B)$ dependence, and obtained a good agreement between the numerical model and the measurement for both the reference and 2LUPS samples.

\section{ACKNOWLEDGMENT}

A.R.I. and A.C.W. acknowledge the financing by the ATOMIS DFF-Research Project which is funded by the Danish Council for Independent Research (project ID DFF - 611100252). 


\section{REFERENCES}

[1] C. Senatore, M. Alessandrini, A. Lucarelli, R. Tediosi, D. Uglietti and Y. Iwasa, "Progresses and challenges in the development of high-field solenoidal magnets based on RE123 coated conductors," Supercond. Sci. Technol., vol. 27, no. 10, 2014, 103001 1-26.

[2] X. Obradors and T. Puig, "Coated conductors for power applications: materials challenges," Supercond. Sci. Technol., vol. 27, no. 4, 2014, 044003 1-17.

[3] Y. Zhang, T.F. Lehner, T. Fukushima, H. Sakamoto and D.W. Hazelton, "Progress in Production and Performance of Second Generation (2G) HTS Wire for Practical Applications," Proceedings of 2013 IEEE International Conference on Applied Superconductivity and Electromagnetic Devices, Beijing, China, 2013, ID3093 557 - 558.

[4] D. Larbalestier, A. Gurevich, D. M. Feldmann and A. Polyanskii, "HighTc superconducting materials for electric power applications," Nature, vol. 414, 2001, 368-377.

[5] M. D. Sumption, E. Lee, C. B. Cobb, P. N. Barnes, T. J. Haugan, J. Tolliver, C. E. Oberly and E. W. Collings, "Hysteretic Loss vs. Filament Width in Thin YBCO Films Near the Penetration Field," IEEE Trans. on Appl. Superc., vol. 13, no. 2, 2003, 3553-3556.

[6] Y. Yanagisawa, Y. Xu, X. Jin, H. Nakagome and H. Maeda, "Reduction of Screening Current-Induced Magnetic Field of REBCO Coils by the Use of Multi-Filamentary Tapes," IEEE Trans. on Appl. Superc., vol. 25, no. 3, 2015, 6603705 1-5.

[7] J. W. J. Carr and C. E. Oberly, "Filamentary YBCO Conductors For AC Applications," IEEE Trans. on Appl. Superc., vol. 9, no. 2, 1999, 14751478.

[8] C. B. Cobb, P. N. Barnes, T. J. Haugan, J. Tolliver, E. Lee, M. Sumption, E. Collings and C. E. Oberly, "Hysteretic loss reduction in striated YBCO," Physica C, vol. 382, 2002, 52-56.

[9] I. Kesgin, G. A. Levin, G. A. Levin and V. Selvamanickam, "Multifilament, copper-stabilized superconductor tapes with low alternating current loss," Appl. Phys. Lett., vol. 103, 2013, 252603 1-3.

[10] S. C. Hopkins, T. B. Mitchell-Williams, D. R. Vanden Bussche, A. Calleja, V. R. Vlad, M. Vilardell, X. Granados, T. Puig, X. Obradors, A. Usoskin, M. Soloviov, M. Vojenčiak, F. Gömöry, I. Van Driessche, M. Bäcker and B. A. Glowacki, "Low AC Loss Inkjet-Printed Multifilamentary YBCO Coated Conductors," IEEE Trans. Appl. Supercond., vol. 26, no. 3, 2016., 6602905 1-5

[11] I. Kesgin, G. Majkic and V. Selvamanickam, "Fully filamentized HTS coated conductor via striation and selective electroplating," Physica C: Supercond., vol. 486, 2013, 43-50.

[12] R. Nast, M. Vojenčiak, E. Demencik, A. Kario, B. Ringsdorf, A. Jung, B. Runtsch, F. Grilli and W. Goldacker, "Influence of laser striations on the properties of coated conductors," J. Phys.: Conf. Ser., vol. 507, 2014, $0220231-5$.

[13] A. C. Wulff, J. H. Lundeman, J. B. Hansen, O. V. Mishin, Y. Zhao, R. Mohajeri and J.-C. Grivel, "A two level undercut-profile substrate for chemical-solution-based filamentary coated conductors," IEEE Trans. on Appl. Superc., vol. 26, no. 3, 2016, 6601604 1-4.

[14] A. C. Wulff, M. Solovyov, F. Gömöry, A. B. Abrahamsen, O. V. Mishin, A. Usoskin, A. Rutt, J. H. Lundeman, K. Thydén, J. B. Hansen and J.-C. Grivel, "Two level undercut-profile substrate for filamentary YBa2Cu3O7 coated conductors," Supercond. Sci. Technol., vol. 28, 2015, Art. ID. 072001

[15] A. Sundaram, Y. Zhang, A. R. Knoll, D. Abraimov, P. Brownsey, M. Kasahara, G. M. Carota, R. Nakasaki, J. B. Cameron, G. Schwab, L. V. Hope, R. M. Schmidt, H. Kuraseko, T. Fukushima and D. W. Hazelton, "2G HTS wires made on $30 \mu \mathrm{m}$ thick Hastelloy substrate," Supercond. Sci. Technol., vol. 29, 2016, 104007 1-6.

[16] THEVA Dünnschichttechnik GmbH, "Datasheet TAPESTAR ${ }^{\mathrm{TM}}$ XL," specs-theva-tapestar-xl-101114, 2014.

[17] K. Higashikawa, D. Uetsuhara, M. Inoue, S. Fujita, Y. Iijima and T. Kiss, "Characterization of Local Critical Current Distribution in Multifilamentary Coated Conductor Based on Reel-to-Reel Scanning Hall-Probe Microscopy," IEEE Trans. Appl. Supercond., vol. 27, 2017, $66030041-4$

[18] X.-F. Li, A. B. Yahia, G. Majkic and V. Selvamanickam, "Scanning Hall Probe Microscopy on Laser Striated Multifilament Coated Conductor," IEEE Trans. on Appl. Superc., vol. 25, no. 3, 2015, 9000404 1-4.

[19] M. Carrera, J. Amorós, X. Obradors, J. Fontcuberta, "A new method of computation of current distribution maps in bulk high-temperature superconductors: analysis and validation," Supercond. Sci. Technol., vol. 16 , no. $10,2003,1187$.
[20] M. Eisterer, "The significance of solutions of the inverse Biot-Savart problem in thick superconductors," Supercond. Sci. Technol., vol. 18, no. 2, 2005, S58.

[21] Hengstberger, F., M. Eisterer, M. Zehetmayer and H. W. Weber, "Assessing the spatial and field dependence of the critical current density in YBCO bulk superconductors by scanning Hall probes," Supercond. Sci. Technol., vol. 22, no. 2, 2009, 025011 .

[22] M. Carrera, J. Amorós, X. Granados, R. Maynou, T. Puig, X. Obradors, "Computation of Current Distribution in YBCO Tapes with Defects Obtained from Hall Magnetic Mapping by Inverse Problem Solution," IEEE Trans. Appl. Supercond., vol. 21, 2011, 3408-3412.

[23] J. Amorós, M. Carrera, X. Granados, "An effective model for fast computation of current distribution in operating HTS tapes from magnetic field measurements in non-destructive testing," Supercond. Sci. Technol., vol. 25, 2012, 104005 1-8.

[24] R. Nakasaki, P. Brownsey, A. Sundaram, Y. Zhang, D. Hazelton, H. Sakamoto, and T. Fukushima, "Progress of 2G HTS Wire Development at SuperPower," Presented at Applied Superconductivity Conference, ASC 2016, 1MOr2A-03, September 4-9, 2016, Denver, CO, USA

[25] V. M. R. Zermeno, A. B. Abrahamsen, N. Mijatovic, B. B. Jensen and M. P. Sørensen, "Calculation of alternating current losses in stacks and coils made of second generation high temperature superconducting tapes for large scale application," J. Appl. Phys., vol. 114, no. 17, 2013, $1739011-8$

[26] C. P. Bean, "Magnetization of High-Field Superconductors," Rev. Mod. Phys., vol. 36, 1964, 31-39.

[27] C. P. Bean, "Magnetization of Hard Superconductors," Phys. Rev. Letters, vol. 8, no. 6, 1962, 250-253.

[28] V. M. R. Zermeño, K. Habelok, M. Stępień and F. Grilli, "A parameterfree method to extract the superconductor's $\mathrm{Jc}(\mathrm{B}, \theta)$ field-dependence from in-field current-voltage characteristics of HTS tapes," Supercond. Sci. Technol., vol. 30, 2017, 034001 1-7.

[29] X.-F. Li, M. Kochat, G. Majkic and V. Selvamanickam, "Critical current density measurement of striated multifilament-coated conductors using a scanning Hall probe microscope," Supercond. Sci. Technol., vol. 29, 2016, 085014 1-10 